\title{
Spectral Problem for Water Flow Glazings
}

\author{
Xabier Romero ${ }^{\mathrm{a}, \mathrm{b}, *}$, Juan A. Hernández ${ }^{\mathrm{b}}$ \\ ${ }^{a}$ Department of Aeronautics, Imperial College London, South Kensington Campus, SW7 2AZ London, UK \\ ${ }^{b}$ Department of Applied Mathematics, School of Aeronautical and Space Engineering, Technical University of Madrid \\ (UPM), Plaza Cardenal Cisneros 3, 28040 Madrid, Spain
}

\begin{abstract}
Water flow glazings are characterized by a water chamber which transports the absorbed solar energy in the glazing. A multilayer water flow glazing comprises glass layers, PVB (Polyvinyl Butyral) layers, coatings, air gaps, and a water layer. Heat absorption from solar radiation depends on the spectral properties of each layer. Energy performance is governed by the optical and radiative properties of the layers. The refractive index and the extinction coefficient define the optical properties of a layer. The optical properties of the water layer are known from the scientific literature (Hale and Querry, 1973). Transmittance and reflectance for every wavelength are measurements supplied by manufacturers and are readily available from the International Glazing Database (IGDB) as a function of wavelength at normal incidence. In this work, a net energy balance radiation model similar to Siegel (1973) is used to solve the spectral problem. A matrix difference equation for a vector of dimension two with boundary conditions is stated to obtain the absorptances of each layer and each interface. The inputs of this set of equations are the transmissivity of each layer, and the transmittance and reflectances of each interface which are based on the refractive index and the extinction coefficient. When these values are not known, an inverse problem is solved to obtain the refractive index and the extinction coefficient of each layer for every wavelength from transmittance and reflectance measurements of the IGDB. For coatings, another inverse problem is formulated to obtain the properties of the coated interface using also transmittance and reflectance measurements of the coated glass pane and the substrate. The outputs of this work are the wavelength-averaged absorptances of each layer, which determine the fractions of the impinging radiation which is absorbed in each layer. The results constitute the inputs of the thermal problem of a water flow glazing.
\end{abstract}

Keywords: water glazing, spectral properties, glass, coating

\footnotetext{
*Corresponding author

Email addresses: xabier.romero-saenz15@imperial.ac.uk (Xabier Romero), juanantonio.hernandez@upm.es (Juan A. Hernández)
} 


\section{Highlights}

- A spectral center-of-glass multi-layer model is described for water flow glazings

- The model is directly applicable as it is based on a public glazing database with manufacturer data

- The formulation retrieves the inputs for thermal calculations of every layer and interface

- The model and the formulation is validated against different tools 


\section{List of symbols}

$T \quad$ : Transmittance of the glazing

$R \quad$ : Reflectance of the glazing

A : Absorptance of the glazing

$T_{0} \quad$ : Transmittance of the glazing at normal incidence angle

$R_{0} \quad$ : Front reflectance of the glazing at normal incidence angle

$R_{0}^{\prime} \quad$ : Back reflectance of the glazing at normal incidence angle

$A^{\prime} \quad$ : Back absorptance of the glazing

$R^{\prime} \quad$ : Back reflectance of the glazing

$t_{i} \quad:$ Transmittance of interface $i$

$r_{i} \quad:$ Front reflectance of interface $i$

$r_{i}^{\prime} \quad$ : Back reflectance of interface $i$

$A_{i} \quad$ : Front absorptance of layer $i$

$A_{i}^{\prime} \quad$ : Back absorptance of layer $i$

$\alpha_{i} \quad:$ Front absorptance of interface $i$

$\alpha_{i}^{\prime} \quad$ : Back absorptance of interface $i$

$n \quad$ : Refractive index

$\rho_{i} \quad:$ Reflectivity of interface $i$

$\tau_{i} \quad:$ Transmissivity of layer $i$

$\theta \quad$ : Angle of incidence

$\lambda \quad$ : Wavelength

$\kappa \quad:$ Extinction coefficient

d : Thickness

$q_{i} \quad$ : Heat flux absorbed in layer $i$

$I_{i} \quad$ : Inward radiosity travelling through the $i$ layer

$J_{i} \quad$ : Outward radiosity travelling through the $i$ layer

$\mathbf{U}_{i} \quad$ : Array of radiosities for layer $i$

$\mathbf{E}_{i} \quad$ : Matrix for the contribution of $\mathbf{U}_{i-1}$ in $\mathbf{U}_{i}$

$\mathbf{F}_{i} \quad$ : Matrix for the contribution of $\mathbf{U}_{i+1}$ in $\mathbf{U}_{i}$

$\mathbf{G}_{i} \quad$ : Fundamental solution of $\mathbf{U}_{i}$ associated to the boundary condition $\mathbf{U}_{0}$

$\mathbf{H}_{i} \quad$ : Fundamental solution of $\mathbf{U}_{i}$ associated to the boundary condition $\mathbf{U}_{N+2}$

$\mathbf{L}_{i} \quad$ : Lower matrix for the $L U$ factorization

$\mathbf{V}_{i} \quad$ : Upper matrix for the $L U$ factorization

$\mathbf{Y}_{i} \quad$ : Auxiliary matrix for the fundamental solution $\mathbf{G}_{i}$

I $\quad: 2 \times 2$ identity matrix

$P V B \quad$ : Polyvinyl Butyral

$I G D B$ : International Glazing Database

$q_{i}^{I} \quad$ : Heat flux absorbed in interface $i$ 


\section{Introduction}

The use of glass is increasingly extending in buildings. Energy efficiency is heavily influenced by the performance of the glazings (Klainsek, 1991). Heat exchange between the building and the exterior affects the energy consumption and HVAC requirements. The properties of the glazing determine the fractions of radiation which are reflected, transmitted and absorbed. Complex configurations can significantly alter the amount of energy absorbed in each layer and impact performance. The distinguishing feature of a water layer in the glazing, requires to accordingly develop models to conduct simulations for the evaluation of the energy performance of buildings.

The distinguishing characteristic of a water flow glazing is an active water chamber layer which transports energy within the glazing. The concept is readily applied to buildings, exemplified by the installation of a water flowing glazing at a building in Carcagente, Spain in 2010. Figure 17 shows the completed status of the installation of the active glazing including the piping for the water flow and its integration into the glass panes. A demonstrator exposed in BAU, Munich in 2017 developed under the InDeWaG project (Industrial Development of Water Flow Glazing) is presented in Figure 1p. Qahtan et al. (2014) applied a flowing water film for building cooling in the tropics showing that with a water layer up to a $70 \%$ of the infrared radiation can be absorbed resulting in an improvement thermal and visual comfort. The main advantages of a water flow glazing are a highly transparent facade, cooling and heating capacity, no need of blind installation, a thin skin glazing (70 $\mathrm{mm}$ compared to the 0.5 to $1 \mathrm{~m}$ of a ventilated facade) and low maintenance. The principal drawback is the complexity of the installation which poses a laborious constructive process and design. The installation costs of a water flowing facade are comparable to a solar panel installation and slightly lower to ventilated facades.

(a)

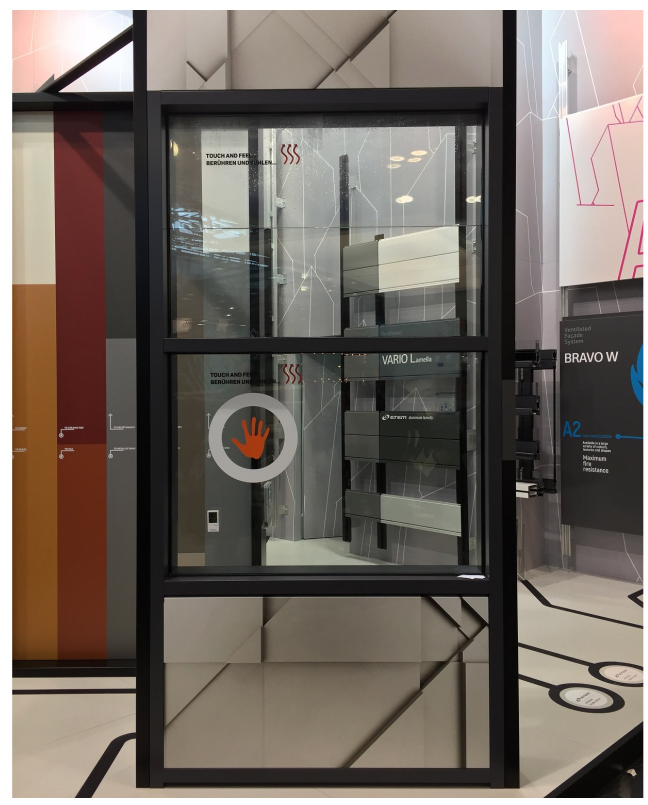

(b)

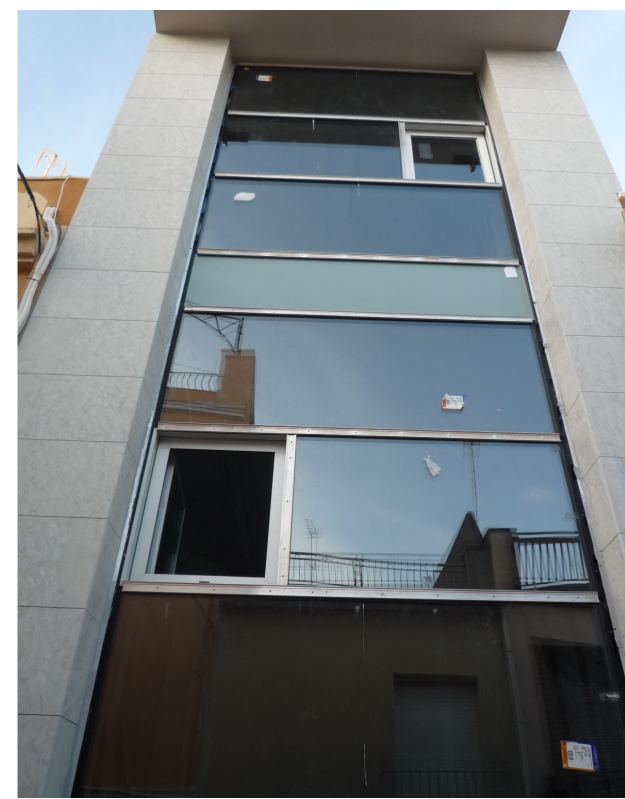

Figure 1: Water flowing glazing examples: (a) InDeWaG project demonstrator at BAU, and (b) completed installation in Carcagente (Spain).

Glazings are required energy efficiency elements rather than mere transparent insulators. While in winter 
solar heat gains should be maximized, in summer they can produce overheating. If the solar heat gain is not controlled dynamically, an advanced glazing can actually be detrimental in terms of overheating problems (Jelle et al. 2012). To overcome this issue, overhangs, blinds or smart glazings are a possible solution. The distinguishing characteristic of a smart glazing is reduction of both heating and cooling demand during the whole year (Bahaj et al. 2008).

Smart glazings can adjust their optical and thermal properties according to climate conditions. Electrochromic glazings (Granqvist, 2008) and liquid crystals (Gardiner et al. 2009) glazings are two examples of smart window technologies. These glazings change the optical properties when a voltage is applied (Granqvist et al. 2009). The glazing switches between clear and colored states. The switching action can be modulated to any intermediate state by means of a voltage regulator. Depending on the electrochromic coating, different values of the visual transmittance can be obtained Assimakopoulos et al. 2007). All electrochromic devices modify the thermal or visual transmittance by modulating the short wave absorptance rather than the short wave reflectance. Hence, these glass panes are heated up as if they were tinted glasses. This drawback obliges to arrange the electrochromic coating in a position which allows to transfer outdoors the absorbed energy, which can not be harvested. Alternative examples include vacuum glazings (Cuce and Cuce, 2016, Fang et al., 2010), low emittance coatings (Fang et al., 2007; Gorgolis and Karamanis, 2016), photovoltaic glazings (Tina et al., 2013, Liao and Xu, 2015) and Phase Change Materials (Silva et al., 2016, Goia, 2012, Goia et al. 2012).

Water flow glazings are a further example of smart glazing (Chow et al., 2011, 2010). This solutions is based on circulating a water flow in a close circuit allowing the thermal solar heat gain to be controlled dynamically. In this case, the water flow does not modify the visual transmittance of the glazing but the temperature of the glass panes which are in contact with the water. By varying the water flow rate, the indoor thermal flux is controlled (Sierra and Hernández, 2017). In this case, the absorbed energy is transported allowing energy harvesting. Hence, electrochromic coating, which modifies the spectral properties as the visual transmittance, can be complemented with water flow glazings to achieve an advanced smart glazing which allows to modulate the internal heat gain and to harvest solar energy.

This paper focuses on the modeling of multilayer water flow glazings. Multi-physics simulations comprise several physic phenomena simultaneously. In these problems, the solution is coupled. In the case of glazings, two representative examples are heat transfer and photovoltaic effect. These complex multiphysics models are readily available (Suárez et al., 2011; Chow et al., 2007) to estimate the performance of advanced glazing configurations. Lechowska (2016) introduced Computational Fluid Dynamics in the analysis of transmittance of a double glazing and Vera et al. (2014) optimized the performance of a domestic hot water system including thermal effects on photovoltaic collectors. Despite the existence of sophisticated tools, a simpler, at the expense of detailed description of the glazing performance, are center-of-glass models (Karlsson et al., 2001, Ismail and Henrıquez, 2003) are an efficient and accurate tool for estimations.

Center-of-glass models require modeling both the environment (exterior and interior conditions) and the physics of the glazing. Exterior conditions, such as weather and incoming radiation (Bird et al., 1983, 
Bird, 1984 Hulstrom et al. 1985), are inputs which depend on the location of the building, and are subject to daily and seasonal fluctuations. An optical and a heat transfer model are required to describe the glazing.

The fact that the wavelengths where radiant heat transfer occurs are longer than those wavelengths where radiation is absorbed (Wright, 1998) enables the treatment of the spectral and the heat transfer problems separately. Sierra and Hernández (2017) stated the applicability of this difference in wavelength to a water flowing glazing. The radiation originated from the sun is comprised with visible and near infrared components, while the long wave radiation emitted is a result from the absorption in the glass panes or water chambers of the sun radiation. Therefore, this contribution of long-wave radiation allows to separate the model for the absorption-conduction problem and the optical problem. The current standards for glazing performance evaluation, ISO 9050 and ISO 15099 use the spectral properties as an independent input to the thermal problem. As a result, the optical computations are independent of the temperatures and only require the optical properties of the layers of the glazing. The spectral model focuses on the calculation of the fractions of incident radiations which are absorbed in each layer and interface. Glazing energy models are based on different methods. Examples of these are the matrix method (Pfrommer et al., 1995, Harbecke, 1986, van Nijnatten, 1994), the ray tracing method (Rubin, 1982; Howell et al., 2010) or the net energy balance method (Edwards, 1977; Siegel, 1973, Shurcliff, 1974; Wijeysundera, 1975, Walton, 1986). These methodologies were extended and validated experimentally in a photovoltaic glazing (Baenas and Machado, 2016; Machado et al., 2016). Heat transfer analysis comprises the absorption-conduction problem in the glass panes and the resulting interaction with the exterior and interior environments.

This work presents an optical center-of-glass model for the water flow glazing founded on the net energy balance method. This approach is shared with the ISO 15099 standard. The nomenclature of the standard is preserved for most of the magnitudes in this paper. The presented model can effectively describe the optical behavior of multilayer glazing including glass layers, PVB layers, coated glass layers, air gaps and water layers. The optical behavior inside each of these layers can be described using the refractive index and the extinction coefficient. Interfaces are characterized through their transmittance, and front and back reflectances. Interfaces can account for the interference effects in the case of coated glass. This set of properties describes the behavior of the glazing for different wavelengths and angles of incidence. Spectral Weight Functions (Gueymard and duPont, 2009, ASTM G173-03, 2012) are used to spectrally average the results for a given radiation spectrum and provide global results of the performance of the glass.

The International Glazing Database (IGDB) includes data for a large number of products by several manufacturers. This database provides transmittance, front and back reflectance at normal incidence as a function of wavelength. These measurements can be used to retrieve the required optical properties for the different constituents of a multilayer glazing.

A methodology to link the model with these measurements for simple configurations is provided in this paper. There is no need for additional measurements for data available in the database, even if thicknesses 
or layout is altered. Therefore, the model is suitable to analyze complex configurations using products existing in the IGDB. Coatings can also be introduced through the interface properties. Coatings are deposited in a glassy layer, substrate, and the measurements include the two constituents. The spectral behavior of coatings depends significantly on the substrate and coating combination. A large amount of these combinations can be obtained from the IGDB. Several models are found in the literature (see, for example, Rubin et al. (1999); Maestre et al. (2007); van Nijnatten (1999)). The ADOPT Project (Hutchins and Ageorges, 1993 Hutchins et al. 2001, Roos et al., 2001) evaluated their accuracy and modeling alternatives for coated glass were developed. Existing measurements available in the IGDB can be re-utilized by means of the methodology from ASHRAE (ASHRAE, 2001). This methodology is currently implemented in Window (Finlayson et al. 1993) and EnergyPlus (DOE, 2010).

The remainder of the paper is structured as follows. In section 2 the spectral problem is established in terms of the energy balance of the interfaces. The resulting problem is stated by a set of difference equations with two boundary conditions. The solution of this difference equation is given in section 3 . The particular form of this solution allows to identify the fractions of radiation incoming exterior and interior radiation which are transmitted, absorbed and reflected at each layer and interface of the glazing. The spectral inputs to the thermal problem are identified in section 4 . The basic input configurations and the connection to available measurements from the IGDB are described in section 5 Validation examples are included in section 6 . Section 7 gathers the benefits and concluding remarks of this model.

\section{Difference equations to solve the spectral problem}

The glazing system comprises $\mathrm{N}$ layers together with the outermost layer $i=0$ and the innermost layer $i=N+1$ as it is shown in figure 2 This set of layers is defined by the interfaces from $i=0$ to $i=N+2$.

We denote by $I_{i}, J_{i}$ the radiation flowing towards the innermost and outermost sides respectively at each interface $i$. Hence, $I_{0}$ represents the exterior solar radiation impinging into the glazing and $J_{N+2}$ the interior radiation. These two values will be considered input data to solve the spectral problem. 


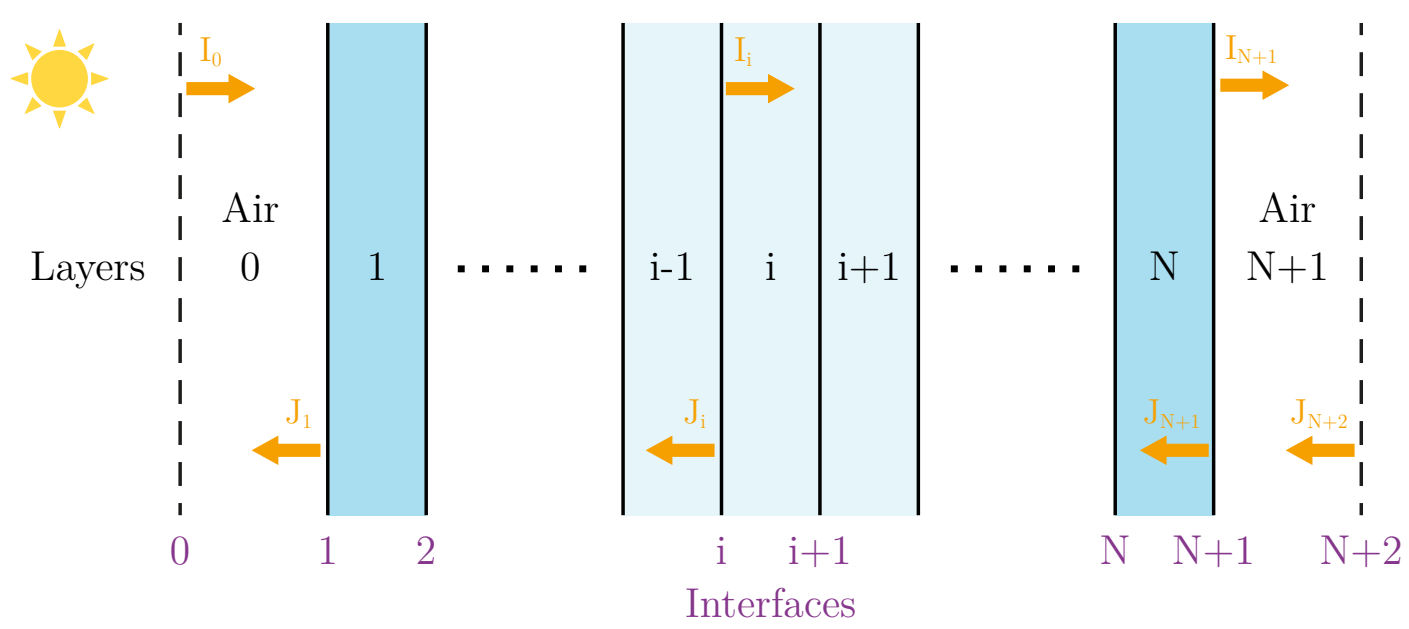

Figure 2: Numbering of layers and interfaces and radiation fluxes $\left(I_{i}, J_{i}\right)$ towards innermost and outermost layers, respectively.

Four optical properties are needed to solve the spectral problem: the transmissivity $\tau_{i}$ of layer $i$, the transmittance $t_{i}$, and the front and back reflectance $r_{i}$ and $r_{i}^{\prime}$ of interface $i$. These input spectral properties are represented in figure 3 and colored in orange to differentiate from the unknowns of the spectral problem.

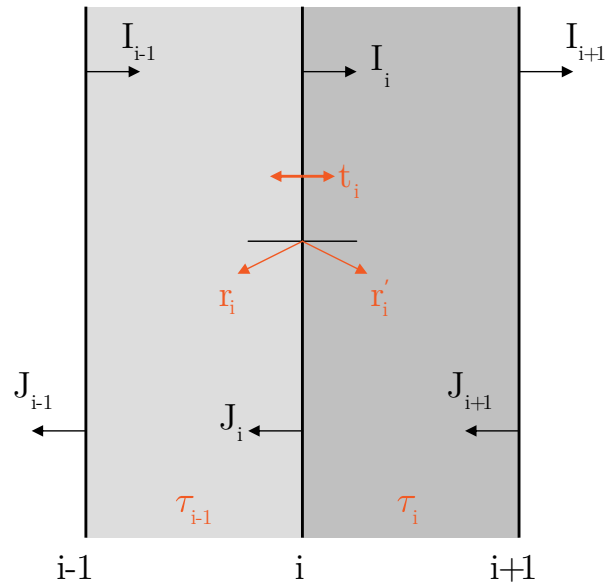

Figure 3: Input optical properties: transmissivity $\tau_{i}$ of layer $i$, transmittance $t_{i}$ and front and back reflectance $r_{i}, r_{i}^{\prime}$ of interface $i$

When solving the electric and magnetic waves traveling into a medium, a complex wave number, known as the complex refractive index, appears naturally. The real part of the index, called the refractive index $n$, indicates the phase velocity. The imaginary part, called the extinction coefficient $\kappa$, measures how this wave is attenuated. By imposing boundary conditions for the magnetic and electric fields at a plane boundary between two different media (Bohren and Huffman, 1983), the reflection and transmission coefficients are determined as functions of the two complex refractive indices which are involved. If the 
media are almost dielectric, the imaginary part (extinction coefficient) $\kappa \ll n$ and the reflectivity of the interface are determined by Fresnel equations. In our work, glass layers, water layers or PVB layers have values of $\kappa$ of $O\left(10^{-3}\right)$ at most and $n$ is close to unity for the visible and NIR wavelengths. Hence, the hypothesis of almost dielectric medium is fulfilled and the expression for the reflectivity simplifies to the one given by Fresnel equations.

If the refractive index and the extinction coefficient for each layer are known, the forward problem consists of determining the transmittance, front and back reflectance of each layer and interface. However, when these optical properties are not known, measurements from the IGDB can be used to solve an inverse problem. This database contains data of glasses, PVB layers and coatings from different manufacturers. For a glass or PVB layer, the transmittance and the reflectance for every wavelength is given as a function of wavelength at normal incidence. The measurements of transmittance and reflectance for each glass or PVB layer, pose the inverse problem of obtaining the refractive index $n$ and the extinction coefficient $\kappa$ of each layer for every wavelength. If no coating is present at the interface $i$, the spectral values $n$ and $\kappa$ allow to determine the transmittance $t_{i}$ and the front and back reflectance of the interface. For coatings, the IGDB provides the transmittance and the reflectance of a specific coating with a substrate. In this case, a different inverse problem is formulated to discount the substrate and to give the transmittance $t_{i}$ and the front and back reflectance $r_{i}, r_{i}^{\prime}$ of the interface where the coating is located. Hence, these optical properties depend on the wavelength of the impinging radiation and vary with the angle of incidence. A detailed procedure to obtain these data will be discussed in section 5. The optical properties of water layer can be found in the scientific literature (Hale and Querry, 1973).

To determine the transmittance of an absorbing material it is necessary to distinguish between thick layers and thin layers. A thin layer has a thickness of the order of the wavelength of the radiation or less. The waves reflected between the interfaces of a thin layer allow to maintain coherence of waves (Stenzel, 2005). The destructive interference leads to attenuation of the electric fields which is understood as absorption. When the thickness of a layer is greater than the coherence length of about $1500 \mathrm{~nm}$ (Pfrommer et al. 1995), disturbances along the path of propagation due to in-homogeneities in the material, disrupt the phases of the waves and destroy coherency. Therefore, for thick layers it is not possible to describe the radiation in terms of electric fields. In this case, the addition of intensities (instead of electric fields) is correct (Stenzel, 2005). Glass, water and PVB layers are considered thick layers in which multiple reflections exist but no interference effects. On the other hand, coatings are considered thin layers and they are characterized by $t, r, r^{\prime}$ which include interference effects.

Since the scattering effects are not considered in the present model, the applicability of the method is limited to non scattering media. The multiple scattering effects can be important for translucent PVB layers, translucent glass layers or water layers with small particles (Jonasz and Fournier, 2011).

The energy balance applied to the interface $i$ can be expressed by the following equations:

$$
\begin{aligned}
I_{i} & =t_{i} \tau_{i-1} I_{i-1}+r_{i}^{\prime} \tau_{i} J_{i+1} \\
J_{i} & =r_{i} \tau_{i-1} I_{i-1}+t_{i} \tau_{i} J_{i+1}
\end{aligned}
$$


A similar formulation can be found in (Siegel, 1973 ISO 15099, 2003, Shurcliff, 1974, Wijeysundera, 1975, Edwards, 1977). The energy balance equations (1) and (2), can be rewritten in matrix form

$$
\left(\begin{array}{c}
I_{i} \\
J_{i}
\end{array}\right)=\left[\begin{array}{ll}
t_{i} \tau_{i-1} & 0 \\
r_{i} \tau_{i-1} & 0
\end{array}\right]\left(\begin{array}{c}
I_{i-1} \\
J_{i-1}
\end{array}\right)+\left[\begin{array}{cc}
0 & r_{i}^{\prime} \tau_{i} \\
0 & t_{i} \tau_{i}
\end{array}\right]\left(\begin{array}{c}
I_{i+1} \\
J_{i+1}
\end{array}\right)
$$

Next, the vector $\mathbf{U}_{i}$, and the matrices $\mathbf{E}_{i}$ and $\mathbf{F}_{i}$ are defined as

$$
\mathbf{U}_{i}=\left(\begin{array}{c}
I_{i} \\
J_{i}
\end{array}\right) \quad \mathbf{E}_{i}=\tau_{i-1}\left[\begin{array}{cc}
t_{i} & 0 \\
r_{i} & 0
\end{array}\right] \quad \mathbf{F}_{i}=\tau_{i}\left[\begin{array}{cc}
0 & r_{i}^{\prime} \\
0 & t_{i}
\end{array}\right]
$$

Introducing these definitions in equation (3), the system can be compactly rewritten as:

$$
\mathbf{U}_{i}=\mathbf{E}_{i} \mathbf{U}_{i-1}+\mathbf{F}_{i} \mathbf{U}_{i+1}, \quad i=1, \ldots N+1
$$

which constitutes a set of difference equations for solving the spectral problem. Applying the energy balance for the $N+1$ interfaces, $N+1$ equations are obtained which involve the vectors $\left\{\mathbf{U}_{i}, \quad i=\right.$ $1, \ldots N+1\}$. Since these vectors have two elements, the problem implies $2(N+1)$ equations with $2(N+2)$ unknowns. The given boundary conditions $I_{0}$ and $J_{N+2}$ complete the number of equations and allow to solve the spectral problem.

The set of equations (1) and (2) can be connected to the electromagnetic theory. The solution for all the layers can also be obtained by superposing electromagnetic signals and imposing continuity boundary conditions at interfaces. In the case of a single layer, the solution given in equation A.1 is similar to (Bohren and Huffman, 1983). When two or more layers are considered a set of continuity boundary conditions should be imposed at each interface to obtain the resulting transmittance of the system, which requires to solve a recurrence relation for the signals of the layers (Heavens, 1991). In the present work, this solution of multiple refections between two or more layers is obtained by means of the recurrence relation given by equations (1) and (2).

In the Internal Radiometric Model (Rubin et al. 1998) used in the present work, the interfaces may be simple or complex. Simple interfaces have no absorption, i.e., a boundary between homogeneous materials like glass or water. Since there is no absorption, $r_{i}=r_{i}^{\prime}, t_{i}=1-r_{i}$, and the reflectivity $r_{i}$ is given by Fresnel equations, see section 5.1. On the contrary, complex interfaces may contain one or more absorbing layers. The internal radiometric model considers the multiple reflections existing inside the different layers but does not consider interference bands. Interference bands may be observed for perfectly monochromatic light. Given that real beams are composed of a finite spread of wavelengths, the model is suitable for glazing analysis. The existing interference effects within the stacks of the thin layers require thin layers to be treated separately.

The problem of multiple reflections inside the coating can be solved (Stenzel, 2005) to give three equations for transmittance $t_{i}$, and the front reflectance $r_{i}$ and back reflectance $r_{i}^{\prime}$ of a coating deposited on a glass substrate. These equations depend on the complex refractive indices of the involved media: air, coating and glass substrate. In this way, the coating may now be considered as a complex interface 
whose calculated properties may be used in the Internal Radiometric Model (Rubin et al., 1998). This approach is motivated by the fact that the complex refractive index of the coatings is not provided by manufacturers. Maestre et al. (2007) introduced a similar formulation to solve the complete inverse problem of characterizing a single layer coating by the complex refractive index using numerical optimization. Besides, the present model includes an additional parameter $\tau_{i}$ to take into account the possibility of absorption within a layer. In the following section, a procedure based on two fundamental solutions is formulated to solve the set of difference equations (5) with the boundary conditions $I_{0}$ and $J_{N+2}$.

\section{Solution of the spectral problem}

The difference equation (5) which governs the spectral problem is a second order equation. When this equation is completed with given values $I_{0}$ and $J_{N+2}$, the problem constitutes a boundary value problem. From the physical point of view, it means that the spectral properties at interface $i$ depend on the interface $i+1$ and interface $i-1$.

It is important to note that equation (5) can be expressed as a linear system of equations where the unknowns are $\left\{\mathbf{U}_{i}, i=1, \ldots N+1\right\}$ and the matrix of the system is tridiagonal with elements which are submatrices of size $2 \times 2$. The $2 \times 2$ identity matrix is denoted by $\mathbf{I}$.

$$
\left(\begin{array}{ccccc}
\mathbf{I} & -\mathbf{F}_{1} & 0 & \cdots & 0 \\
-\mathbf{E}_{2} & \mathbf{I} & -\mathbf{F}_{2} & \ddots & \vdots \\
0 & \ddots & \ddots & \ddots & 0 \\
\vdots & \ddots & -\mathbf{E}_{N} & \mathbf{I} & -\mathbf{F}_{N} \\
0 & \cdots & 0 & -\mathbf{E}_{N+1} & \mathbf{I}
\end{array}\right)\left(\begin{array}{c}
\mathbf{U}_{1} \\
\vdots \\
\vdots \\
\vdots \\
\mathbf{U}_{N+1}
\end{array}\right)=\left(\begin{array}{c}
\mathbf{E}_{1} \mathbf{U}_{0} \\
0 \\
\vdots \\
0 \\
\mathbf{F}_{N+1} \mathbf{U}_{N+2}
\end{array}\right)
$$

Since the problem is linear, the solution can be expressed by a linear combination of two fundamental solutions associated to each boundary condition: (i) $\mathbf{G}_{i}$ solution for $\mathbf{U}_{0}=\mathbf{I}$ and $\mathbf{U}_{N+2}=\mathbf{0}$ and (ii) $\mathbf{H}_{i}$ solution for $\mathbf{U}_{0}=\mathbf{0}$ and $\mathbf{U}_{N+2}=\mathbf{I}$. By superposing these two fundamental solutions, the general solution of the spectral problem is expressed:

$$
\mathbf{U}_{i}=\mathbf{G}_{i} \mathbf{U}_{0}+\mathbf{H}_{i} \mathbf{U}_{N+2} .
$$

To obtain these two fundamental solutions, a $L U$ decomposition (Press et al., 2007) of the matrix of the system is accomplished taking into account that the matrix is tridiagonal. The matrix is factorized into a lower triangular matrix and an upper triangular matrix, where both matrices are bidiagonal

$$
\left(\begin{array}{ccccc}
\mathbf{I} & 0 & \ldots & \ldots & 0 \\
-\mathbf{L}_{2} & \mathbf{I} & 0 & \ldots & 0 \\
0 & \ddots & \ddots & \ddots & \vdots \\
\vdots & \ddots & -\mathbf{L}_{N} & \mathbf{I} & 0 \\
0 & \ldots & 0 & -\mathbf{L}_{N+1} & \mathbf{I}
\end{array}\right)\left(\begin{array}{ccccc}
\mathbf{V}_{1} & -\mathbf{F}_{1} & 0 & \ldots & 0 \\
0 & \mathbf{V}_{2} & -\mathbf{F}_{2} & \ddots & \vdots \\
\vdots & \ddots & \ddots & \ddots & 0 \\
0 & \ldots & 0 & \mathbf{V}_{N} & -\mathbf{F}_{N} \\
0 & \ldots & \ldots & 0 & \mathbf{V}_{N+1}
\end{array}\right)
$$


Identifying the elements of the matrix of (6) with the elements of resulting product of equation (8), a recurrence relation for the upper and lower matrices is obtained:

$$
\begin{array}{rlrl}
\mathbf{L}_{i} & =\mathbf{E}_{i} \mathbf{V}_{i-1}^{-1}, & i & =2, \ldots N+1 \\
\mathbf{V}_{i}=\mathbf{I}-\mathbf{L}_{i} \mathbf{F}_{i-1}, & i & =2, \ldots N+1
\end{array}
$$

with $\mathbf{V}_{1}=\mathbf{I}$.

Once the matrix is factorized, the two fundamental solutions are obtained by forward and backward substitution. The forward substitution for the $\mathbf{G}_{i}$ solution is:

$$
\mathbf{Y}_{i}=\mathbf{L}_{i} \mathbf{Y}_{i-1}, \quad i=2, \ldots N+1
$$

with $\mathbf{Y}_{1}=\mathbf{E}_{1}$. In the same manner, the forward substitution for the $\mathbf{H}_{i}$ solution gives: $\mathbf{Y}_{i}=0, i=1 \ldots N$ and $\mathbf{Y}_{N+1}=\mathbf{F}_{N+1}$.

The final backward substitution involves the upper triangular matrix and gives the following recurrence relationships for the two fundamental solutions:

$$
\begin{aligned}
& \mathbf{G}_{i}=\mathbf{V}_{i}^{-1}\left(\mathbf{Y}_{i}+\mathbf{F}_{i} \mathbf{G}_{i+1}\right), \quad i=N, \ldots 1 \\
& \mathbf{H}_{i}=\quad \mathbf{V}_{i}^{-1} \mathbf{F}_{i} \mathbf{H}_{i+1}, \quad i=N, \ldots 1
\end{aligned}
$$

with $\mathbf{G}_{N+1}=\mathbf{V}_{N+1}^{-1} \mathbf{Y}_{N+1}$ and $\mathbf{H}_{N+1}=\mathbf{V}_{N+1}^{-1} \mathbf{F}_{N+1}$.

It can be shown that matrices $\mathbf{G}_{i}$ and $\mathbf{H}_{i}$ respond to this shape:

$$
\mathbf{G}_{i}=\left[\begin{array}{cc}
a_{i} & 0 \\
b_{i} & 0
\end{array}\right], \quad \mathbf{H}_{i}=\left[\begin{array}{cc}
0 & c_{i} \\
0 & d_{i}
\end{array}\right]
$$

and consequently, the two components of the solution expressed by equation (7) yield:

$$
\begin{aligned}
& I_{i}=a_{i} I_{0}+c_{i} J_{N+2} \\
& J_{i}=b_{i} I_{0}+d_{i} J_{N+2}
\end{aligned}
$$

\section{Spectral properties obtained from the solution}

Once the spectral problem is solved, the global spectral properties: transmittance $(T)$, front and back reflectance $\left(R, R^{\prime}\right)$ can be obtained from equations 16 and 17 .

The transmittance $T$ is defined as the fraction of the impinging radiation $I_{0}$ which is transmitted, and the back reflectance $R^{\prime}$ is the proportion of the interior radiation $J_{N+2}$ which is reflected in the interior interface. Particularizing equation 16 for the interface $i=N+1$ :

$$
I_{N+1}=\underbrace{a_{N+1}}_{T} I_{0}+\underbrace{c_{N+1}}_{R^{\prime}} J_{N+2}
$$


In the same way, the front reflectance $R$ is the fraction of the impinging radiation $I_{0}$ which is reflected in the outer interface, and the back transmittance $T^{\prime}$ is the fraction of the interior radiation $J_{N+2}$ which is transmitted. Equation 17 at interface $i=1$ yields:

$$
J_{1}=\underbrace{b_{1}}_{R} I_{0}+\underbrace{d_{1}}_{T^{\prime}} J_{N+2}
$$

By this way, the global spectral properties are identified with the following coefficients of the solution:

$$
T=a_{N+1}, \quad T^{\prime}=d_{1}, \quad R=b_{1}, \quad R^{\prime}=c_{N+1}
$$

The front absorptance $A_{i}$ for layer $i$ is defined as the fraction of radiation $I_{0}$ which is absorbed. In the same manner, the back absorptance $A_{i}^{\prime}$ is the fraction of absorbed radiation $J_{N+2}$. Hence, the absorbed heat flux $q_{i}$ in layer $i$ can be expressed as:

$$
q_{i}=A_{i} I_{0}+A_{i}^{\prime} J_{N+2}
$$

Figure 4 shows a diagram for the computation of radiation flux which is absorbed in a layer $i$. There are two fluxes which cross the layer $i$. The outer radiation $I_{i}$ comes from the left side and the inner radiation $J_{i+1}$ from the right side. Accounting for the transmissivity $\tau_{i}$ of the layer $i$, the transmitted radiation fluxes are $\tau_{i} I_{i}$ and $\tau_{i} J_{i+1}$. The energy which is not transmitted is absorbed, thus the resulting net absorbed heat flux $q_{i}$ in layer $i$ is:

$$
q_{i}=\left(1-\tau_{i}\right) I_{i}+\left(1-\tau_{i}\right) J_{i+1}
$$

Expressing $I_{i}$ and $J_{i+1}$ as a function of $I_{0}$ and $J_{N+2}$ using equations (16) and (17), the absorbed heat flux is obtained:

$$
q_{i}=\underbrace{\left(1-\tau_{i}\right)\left(a_{i}+b_{i+1}\right)}_{A_{i}} I_{0}+\underbrace{\left(1-\tau_{i}\right)\left(c_{i}+d_{i+1}\right)}_{A_{i}^{\prime}} J_{N+2}
$$

Front absorptance $A_{i}$ and back absorptance $A_{i}^{\prime}$ can be identified in equation (23) by means of the previous definition of front and back absorptance of equation (21), to yield:

$$
\begin{array}{ll}
A_{i}=\left(1-\tau_{i}\right)\left(a_{i}+b_{i+1}\right) & i=1, \ldots N \\
A_{i}^{\prime}=\left(1-\tau_{i}\right)\left(c_{i}+d_{i+1}\right) & i=1, \ldots N
\end{array}
$$




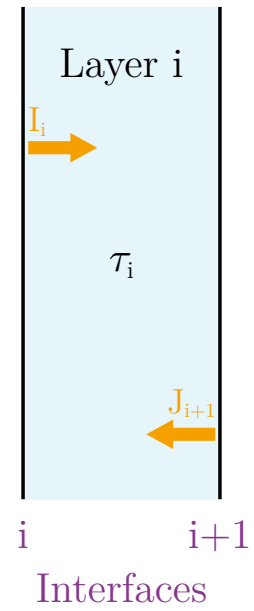

Figure 4: Absorbed energy of a layer with transmissivity $\tau_{i}$ subject to a left radiation flux $\left(I_{i}\right)$ and right radiation flux $\left(J_{i+1}\right)$.

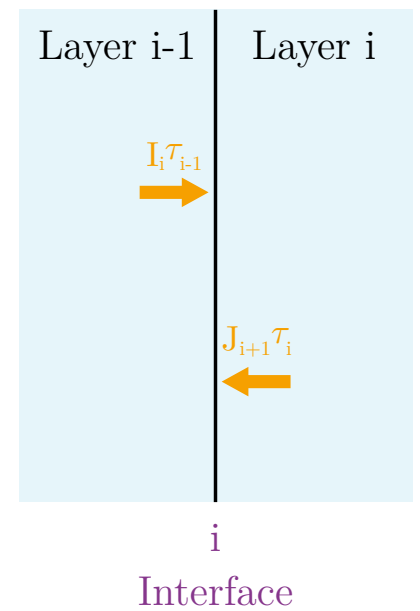

Figure 5: Absorbed energy at interface $i$. Energy balance of impinging left radiation flux $\left(\tau_{i-1} I_{i}\right)$ and right radiation flux $\left(\tau_{i} J_{i+1}\right)$ at interface $i$.

Radiation fluxes can also be absorbed in the interfaces. Figure 5 shows a scheme for the calculation of the heat flux $q_{i}^{I}$ absorbed at interface $i$. There are two radiation fluxes that can be absorbed. The first one is associated to the incoming radiation $I_{i}$. From this radiation only part of it reaches the interface: $\tau_{i-1} I_{i}$. The fraction absorbed by the interface corresponds to the radiation which is not reflected nor transmitted: $\left(1-t_{i}-r_{i}\right) \tau_{i-1} I_{i}$. The second one relates to the incoming radiation $J_{i+1}$. In this case, the amount of radiation reaching the interface is $\tau_{i} J_{i+1}$. In this case, the remaining part not being reflected nor transmitted is $\left(1-t_{i}-r_{i}^{\prime}\right) \tau_{i} J_{i+1}$. Adding the two contributions the heat flux corresponds to

$$
q_{i}^{I}=\left(1-t_{i}-r_{i}\right) \tau_{i-1} I_{i}+\left(1-t_{i}-r_{i}^{\prime}\right) \tau_{i} J_{i+1}
$$

Interface front absorptance $\alpha_{i}$ and interface back absorptance $\alpha_{i}^{\prime}$ for an interface $i$ are defined as the fraction of the incident radiation from the exterior and the interior absorbed in the interface, respectively. 
The absorbed heat flux $q_{i}^{I}$ in the interface $i$ is:

$$
q_{i}^{I}=\alpha_{i} I_{0}+\alpha_{i}^{\prime} J_{N+2}
$$

Given that $I_{i}$ and $J_{i+1}$ are expressed in terms of $I_{0}$ and $J_{N+2}$ using equations (16) and (17), the absorbed heat flux in the interface can be written:

$$
\begin{aligned}
q_{i}^{I} & =\underbrace{\left[\left(1-t_{i}-r_{i}\right) \tau_{i-1} a_{i-1}+\left(1-t_{i}-r_{i}^{\prime}\right)\left(1-\tau_{i}\right) b_{i+1}\right]}_{\alpha_{i}} I_{0}+ \\
& +\underbrace{\left[\left(1-t_{i}-r_{i}\right) \tau_{i-1} c_{i-1}+\left(1-t_{i}-r_{i}^{\prime}\right) \tau_{i} d_{i+1}\right]}_{\alpha_{i}^{\prime}} J_{N+2}
\end{aligned}
$$

For each interface, interface front absorptance $\alpha_{i}$ and interface back absorptance $\alpha_{i}^{\prime}$ can be identified comparing equations 27 and 28 :

$$
\begin{array}{rlrl}
\alpha_{i}=\left(1-t_{i}-r_{i}\right) \tau_{i-1} & a_{i-1}+\left(1-t_{i}-r_{i}^{\prime}\right) \tau_{i} b_{i+1} & i=1, \ldots N+1 \\
\alpha_{i}^{\prime}=\left(1-t_{i}-r_{i}\right) \tau_{i-1} c_{i-1}+\left(1-t_{i}-r_{i}^{\prime}\right) \tau_{i} d_{i+1} & i=1, \ldots N+1
\end{array}
$$

\section{IGDB basic input configurations to the spectral problem}

The energy balance equations (1) and (2) which govern the spectral problem involve four optical properties: the transmissivity $\tau_{i}$ of layer $i$, the transmittance $t_{i}$, and the front and back reflectance $r_{i}$ and $r_{i}^{\prime}$ of interface $i$. These properties are a function of the wavelength and the angle of incidence of the impinging radiation.

This section details the procedure to derive the required optical properties in the model $\left(\tau_{i}, t_{i}, r_{i}, r_{i}^{\prime}\right)$ using the measurements available from the IGDB. The values obtained using this procedure can be replaced in the resolution of the spectral problem in case a preferred source of data or measurements is available. The database provides transmittance $T_{0}$, front and back reflectance $R_{0}, R_{0}^{\prime}$ at normal angle of incidence for every wavelength. The problem of a single layer with two interfaces is first considered to set the background to connect the measurements from the IGDB and the optical properties required in the model. Different problems arise for uncoated glass and PVB layers (subsection 5.1, and coated glass (subsection 5.2). The resulting inverse problems, which provide the optical properties required for our model, are detailed in AppendixA and AppendixB respectively.

In this section, energy balances are applied at the interfaces. This approach is similar to the reflection and transmission at a plane boundary (Bohren and Huffman, 1983) and (Heavens, 1991). Extended alternatives to this procedure are the ray tracing technique, see the commercial tool (Corporation, 2008), where the interaction between rays and the boundaries is not predefined. A comprarison on glass samples with applied films by Asdrubali and Baldinelli 2009) concluded that the estimations are correct by both approaches. 


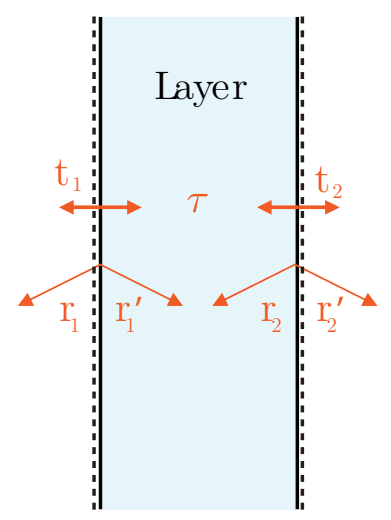

Figure 6: Generic layer of transmissivity $\tau$ with left and right interfaces defined by their spectral properties $: t_{1}, t_{2}, r_{1}, r_{2}, r_{1}^{\prime}, r_{2}^{\prime}$.

A generic layer of transmissivity $\tau$ with left and right interfaces of transmittances $t_{1}, t_{2}$, and front and back reflectances $r_{1}, r_{2}, r_{1}^{\prime}, r_{2}^{\prime}$ is shown in figure6 6 Particularizing equations (1) and (2) for a single layer, the solution to this forward problem is obtained by means of equation 200. The transmittance, front and back reflectance obtained coincide with ISO 9050 and EN 410:2011 standards:

$$
\begin{aligned}
T & =t_{1} t_{2} \tau+\frac{t_{1} t_{2} \tau^{3} r_{1}^{\prime} r_{2}}{1-r_{2} r_{1}^{\prime} \tau^{2}}, \\
R & =r_{1}+\frac{t_{1}^{2} \tau^{2} r_{2}}{1-r_{2} r_{1}^{\prime} \tau^{2}}, \\
R^{\prime} & =r_{2}^{\prime}+\frac{t_{2}^{2} \tau^{2} r_{1}^{\prime}}{1-r_{2} r_{1}^{\prime} \tau^{2}} .
\end{aligned}
$$

It is known that the resulting inverse problem, see equations A.3 A.6, is ill-conditioned giving rise to large errors. Hence, if the complex refractive index of some layer is available, the authors propose to use it directly to obtain the reflectivity of the interface and the transmissivity of the layer. Besides, in order to quantify the committed error, some validation should be carried out. However, sometimes manufacturers claim the use of their special glass arguing that the spectral properties are different to other competitors. The IGDB comprises complete measurements provided by manufacturers. This file comprises the transmittance, the front and the back reflectance of a slab surrounded by air. In the present methodology, we achieve the use of any of these specific products by solving the inverse problem using the data provided by the IGDB. In the same way, some solar coatings or low emissivity coatings are well-kept secrets and the only information provided by manufacturers is the transmittance and reflectance of the coating deposited on substrate. In this case, the inverse problem needs to be solved. The same problem is encountered with solar PVB layers. To minimize errors associated to the inverse problem, and whenever the refractive index $n$ of a material such as soda-lime silica glass is a well known property, a preferable method is to solve $\kappa$ directly from $T$, avoiding the measurement of $R$ which presents a higher uncertainty (Rubin et al. 1998). 


\subsection{Uncoated glass and PVB layers}

Uncoated glass layers and PVB can be defined the refractive index $n$ and the extinction coefficient $\kappa$, frequently referred to as the complex refractive index $n+i \kappa$. Using these two properties the transmissivity $\tau_{i}$ of the $i$-th layer and the properties of the $i$-th interface can be determined $\left(t_{i}, r_{i}, r_{i}^{\prime}\right)$ through the reflectivity $\rho_{i}$. For a single glass layer in air, the transmittance $T$, front and back reflectance $R, R^{\prime}$ are calculated. The IGDB provides the values of $T, R, R^{\prime}$ of a single glass layer in air at normal incidence. In order to describe the $i$-th layer and the $i$-th interface $n$ and $\kappa$ need to be determined using the data available from the IGDB. The inverse problem in this case consists of calculating $n$ and $\kappa$ as functions of the wavelength from the measurements of $T$ and $R$ of a single slab, reversing the steps indicated previously. This procedure is shown graphically in figure 7 . The detailed procedure to determine $n$ and $\kappa$ from IGDB data is indicated in AppendixA.

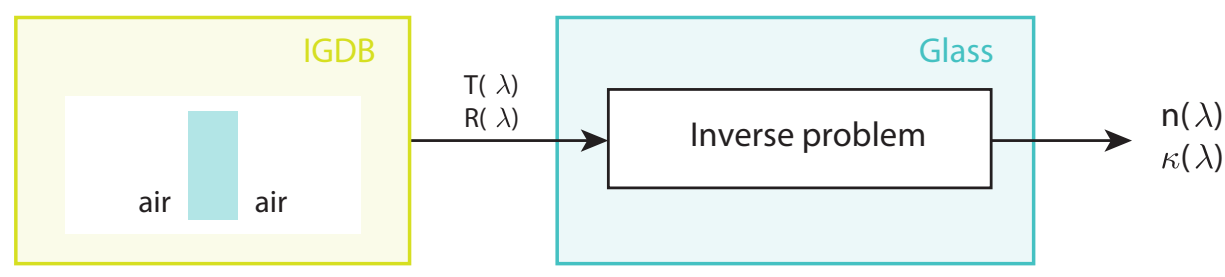

Figure 7: Procedure to solve the inverse problem of an uncoated glass.

Assuming that $n$ and $\kappa$ are known (or calculated using AppendixA the required $\tau_{i}, t_{i}, r_{i}, r_{i}^{\prime}$ are determined. At the interface of the layer, the sum of the incident, reflected and absorbed radiation is equal to one $(t+r+\alpha=1)$. Since there is no coating, no radiation is absorbed $(\alpha=0)$ and the fraction of the incident radiation which is reflected is the reflectivity of the interface, $\rho=r$. Consequently, the transmissivity of the interface is $t=1-\rho$. Note that $r^{\prime}$ is equal to $\rho$ due to the symmetry of the problem.

To take into account the variations of the spectral properties for different angles of incidence, it is necessary to calculate the angle of incidence in each layer. The angle of incidence $\theta_{i+1}$ at a layer $i+1$, using Snell's Law, is:

$$
\theta_{i+1}=\sin ^{-1}\left(\frac{n_{i}}{n_{i+1}} \sin \theta_{i}\right)
$$

where $\theta_{i}$ is the angle measured from the normal of the interface $i$. The transmissivity of layer $i$ is a function of the wavelength $\lambda$ and the angle of incidence $\theta_{i}$ (Rubin et al. 1998):

$$
\tau_{i}(\lambda, \theta)=\exp \left(-\frac{4 \pi \kappa_{i} d_{i}}{\lambda \cos \theta_{i}}\right)
$$

where $d_{i}$ is the thickness and $\kappa_{i}$ the extinction coefficient of layer $i$. The reflectivity for a generic interface $i$ is calculated using the Fresnel equations (Rubin, 1982):

$$
\rho_{i}(\lambda, \theta)=\frac{1}{2}\left[\left(\frac{n_{i-1} \cos \theta_{i-1}-n_{i} \cos \theta_{i}}{n_{i-1} \cos \theta_{i-1}+n_{i} \cos \theta_{i}}\right)^{2}+\left(\frac{n_{i} \cos \theta_{i-1}-n_{i-1} \cos \theta_{i}}{n_{i} \cos \theta_{i-1}+n_{i-1} \cos \theta_{i}}\right)^{2}\right]
$$

Equations 34 - 36 provide the necessary values for the uncoated glass, PVB and water layers, as $r_{i}=r_{i}^{\prime}=\rho_{i}$ and $t_{i}=1-r_{i}$. 


\subsection{Coated glass}

The coating can be characterized by a transmittance $t$ and reflectance $r$ of the of the interface, similarly to the electromagnetic theory of wave propagation (Bohren and Huffman, 1983). This formulation results from the electromagnetic theory and is derived using Fresnel's transmission and reflection coefficients (Heavens, 1991; Stenzel, 2005), as indicated in section 2.

When considering coated glass layers, transmittance $T$, front and back reflectance $R, R^{\prime}$ are obtained for a specific coated glass layer from the IGDB. Spectral properties of the substrate $T_{0}, R_{0}, R_{0}^{\prime}$ are also known from the IGDB. It is considered that there is only one coating at the left interface of the glass layer. To take into account the variations of different angles of incidence, a regression fit (ASHRAE, 2001) is used to calculate the angular properties $T(\theta), R(\theta), R^{\prime}(\theta)$ of coated glass from properties at normal incidence $T(0), R(0), R^{\prime}(0)$. The correlations are based on polynomials of the cosine of the angle of incidence:

$$
\begin{aligned}
& T(\theta)=T(\theta=0) \sum_{k=0}^{4} a_{k}(\cos \theta)^{k} \\
& R(\theta)=R(\theta=0) \sum_{k=0}^{4} b_{k}(\cos \theta)^{k}
\end{aligned}
$$

where the $a_{k}$ and $b_{k}$ coefficients are defined in ASHRAE (2001). The proposed correlation can be replace or substituted by measurements. Once the inverse problem of the substrate is solved, $n$ and $\kappa$ are obtained as functions of the wavelength. The dependence of the optical properties of the substrate with the angle of incidence is determined as in section 5.1. With these considerations, the inverse problem is solved in AppendixB to give the spectral properties of the coated interface $t, r, r^{\prime}$ as a function of the angle of incidence for every wavelength. This procedure is sketched in figure 8 .

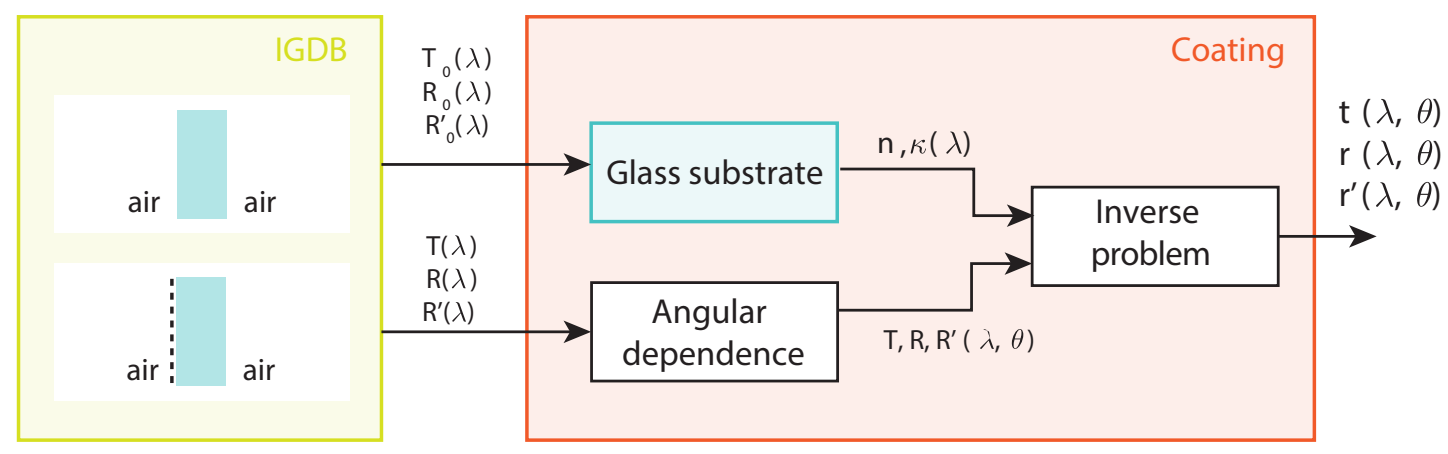

Figure 8: Procedure to solve the inverse problem of a coated glass.

\section{Validation}

In this section various examples are included in order to validate the model presented. The validation was accomplished using Optics and Window (Finlayson et al. 1993) software tools. These tools, together with EnergyPlus, were developed by Lawrence Berkeley National Laboratory dependent on the United 
States Department of Energy. Window software shares the methodology with EnergyPlus, which is a well-established and accepted tool for building analysis in general, and glazing in particular. These tools have different uses. Optics is intended to analyze the spectral properties of glazing systems at normal incidence. Window allows to simulate the performance of a window including frames, dividers and environmental conditions. EnergyPlus simulates the energy performance of complete buildings.

Numerical simulations were conducted in order to validate the method. Four different configurations were examined and compared: a single glass pane, a water layer between two glass panes, a PVB between two different glass panes, and a double glazing with a coated glass pane. Spectral and angular results are provided. Angular results were obtained using the iluminant from the ISO 9050 standard to integrate over the wavelength. Measured data was extracted from the IGDB. The results obtained using the method presented are compared to the ones obtained with Optics for the spectral results and Window for the angular behavior.

Configuration 1 is a single layer of uncoated glass embedded in air of Planilux $6 \mathrm{~mm}$ glass. The inverse problem given by equations A.3 - A.6 of an uncoated glass (see figure 7 at a zero angle of incidence is solved to determine the optical properties $n$ and $\kappa$ and quantify the error introduced by the ill-conditioned inverse problem. The transmittance and reflectance obtained are then recalculated using equations (31) (36). The recalculated values are compared to the measurements in order to quantify the error introduced by the ill-conditioned inverse problem. The transmittance and reflectance as a function of the wavelength using the model and Optics are plotted in figure 9. The results predicted by the model match the results from Optics. The error between the two calculated transmittances is two orders of magnitude smaller than the measurement.

Configuration 2 is a double glazing comprised of a first layer of coated glass embedded in air (Saint Gobain Glass Cool-Lite SKN 154 6mm coated glass on a Planilux 6mm substrate), a 16 mm air chamber and third layer of Planilux 6mm. In this case the inverse problem of a coated glass proposed in figure 8 at a zero angle of incidence is verified. Transmittance, front and back reflectance as a function of a wavelength are represented in figure 10 Results from the model coincide with Optics. The angular behavior of the properties can be observed in figure 11. The higher differences occur at angles between 60 and 80. Regardless of the model, this range of angles is where higher errors occur (Roos et al. 2001$)$. Configuration 3 is a $16 \mathrm{~mm}$ water layer embedded between two $6 \mathrm{~mm}$ Planilux glass panes. Transmittance and reflectance as a function of wavelength are plotted in figure 12 The comparison used in Optics was created as a laminated glass similar to a PVB layer since the software does not include water layers. The specific procedure to generate the layers of the laminate for Optics using Window as described in Section 12 of Sierra and Hernández (2017) was utilized. The optical properties of water were extracted from (Hale and Querry, 1973). Results obtained with both models coincide.

Configuration 4 is a clear PVB layer from Eastman Chemical Company between a Planilux $10 \mathrm{~mm}$ (left) and a $4 \mathrm{~mm}$ glass pane (right). The results shown in figure 13 indicate that the model represents adequately the results. 
The results shown in this section present good agreement with the results provided by the accepted simulation tools.

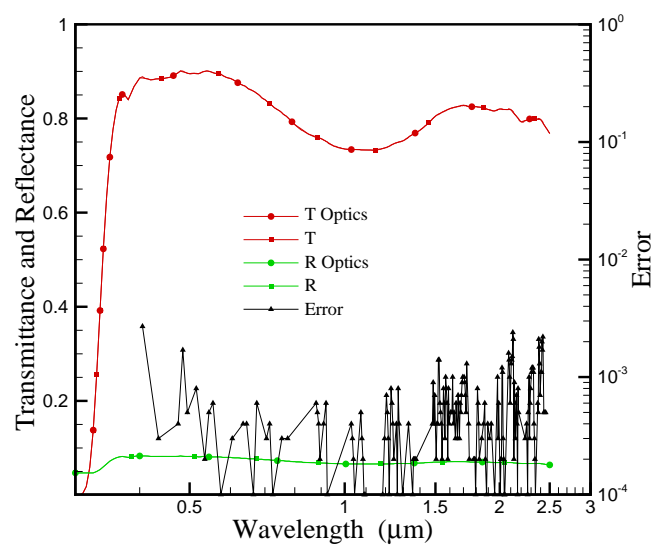

Figure 9: Transmittance, reflectance and error in transmittance of a $6 \mathrm{~mm}$ Planilux glass pane (Configuration 1) as a function of wavelength calculated using the model and Optics.

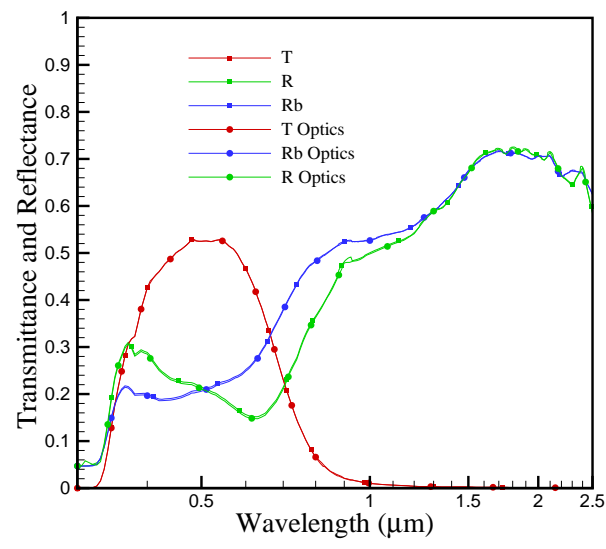

Figure 10: Transmittance and reflectance of a Saint Gobain Glass Cool-Lite SKN $1546 \mathrm{~mm}$ coated glass pane, a $16 \mathrm{~mm}$ air chamber, and a $6 \mathrm{~mm}$ Planilux glass pane (Configuration 2) as a function of wavelength calculated using the model and Optics. 


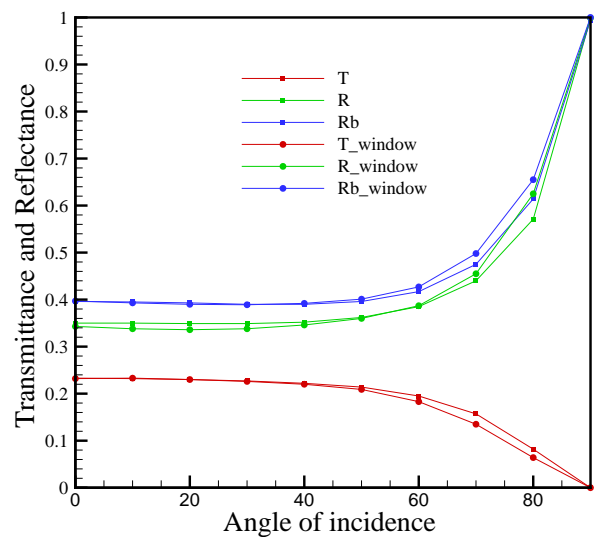

Figure 11: Transmittance and reflectance of a Saint Gobain Glass Cool-Lite SKN $1546 \mathrm{~mm}$ coated glass pane, a 16 mm air chamber, and a $6 \mathrm{~mm}$ Planilux glass pane (Configuration 2) as a function of the angle of incidence calculated using the model and Window.

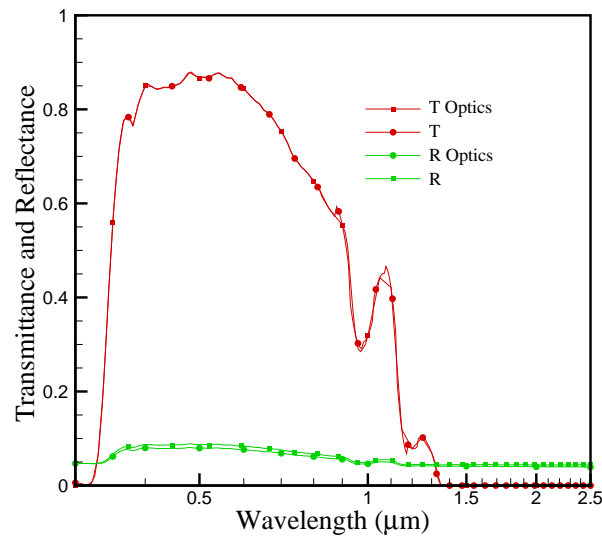

Figure 12: Transmittance and reflectance of a $16 \mathrm{~mm}$ water layer between two 6mm Planilux glass panes (Configuration 3) as a function of wavelength calculated using the model and Optics. 


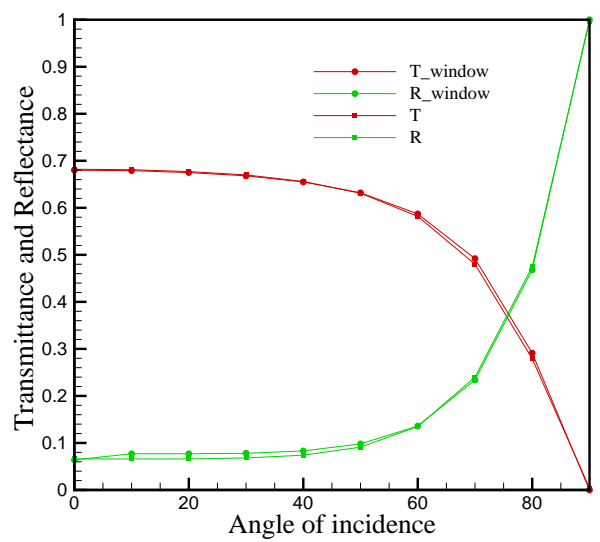

Figure 13: Transmittance and reflectance of a PVB layer Eastman Chemical Company between a Planilux glass pane of $10 \mathrm{~mm}$, left, and a Planinux glass pane of $4 \mathrm{~mm}$, right, (Configuration 4) as a function of the angle of incidence calculated using the model and Window.

\section{Conclusions}

A comprehensive approach to the spectral problem in glazings using a center-of-glass model is detailed in this paper. The described methodology gathers all the necessary elements to simulate water flow glazings in complex configurations including glass layers, PVB, coated glass, and a water layer.

A structured building block approach is presented for the different physics involved in the problem. Examples for each block are provided based on literature and existing simulation tools. The flexibility of the model allows to substitute each block by a distinct one which can be more suitable for a certain application and increase the accuracy of the model. A new solution method for the net energy balance is proposed. The solution is suitable for computer implementation, and can be embedded in a more sophisticated simulation tool for buildings. Numerical results and the validation with well-established software are presented. The agreement in the results indicates that this model is suitable to describe the spectral behavior of several water flow glazing configurations.

Absorptions in each layer and interface are determined for a given configuration. These magnitudes are the inputs to the thermal problem and significantly affect the performance of the installation. Particularly for water flow glazings, heat transfer determines the sizing of the system as a whole. The configuration of the glass, in terms of materials and thicknesses, has an impact on the overall performance of the system. Therefore, it is desirable to simulate a variety of configurations from available measurements for each layer. The connection between the model and measurements at normal incidence using spectrophotometry has been described. These type of measurements are available and accessible for a wide range of materials in the IGDB. Using the approach presented in this paper, the testing of several configurations using reliable data can be simulated without requiring additional measurements.

Further work includes the use of this model combined with a heat transfer model and the description of the behavior of water flow glazings including the heat pump and heat exchanger. The influence of different 
models within this framework is also a subject of further study, particularly for complex configurations including coated layers. It is also an objective to benchmark the performance predicted with these models for long periods of time for a water flow glazing including a more detailed description of window elements.

\section{Acknowledgments}

The authors would like to express their gratitude to Manuel Pena for the insightful discussions and contributions to this paper and to Belén Moreno for providing the photographs from the installation at Carcagente and BAU. Authors thank program Horizon 2020-EU.3.3.1: Reducing energy consumption and carbon footprint by smart and sustainable use, project Ref. 680441 InDeWaG : Industrialized Development of Water Flow Glazing Systems, for financial support for this work.

\section{References}

Asdrubali, F., Baldinelli, G., 2009. Theoretical modelling and experimental evaluation of the optical properties of glazing systems with selective films $2,75-84$.

ASHRAE, 2001. Fundamentals. American Society of Heating, Refrigerating and Air Conditioning Engineers, Atlanta 111.

Assimakopoulos, M., Tsangrassoulis, A., Santamouris, M., Guarracino, G., 2007. Comparing the energy performance of an electrochromic window under various control strategies. Building and Environment 42, 2829 - 2834. doi:http://dx.doi.org/10.1016/j.buildenv.2006.04.004

ASTM G173-03, 2012. Standard tables for reference solar spectral irradiances: Direct normal and hemispherical on $37^{\circ}$ tilted surface .

Baenas, T., Machado, M., 2016. Optical model for multilayer glazing systems: Application to laminated glass and photovoltaic modules. Solar Energy 125, 256 - 266. doi/http://dx.doi.org/10.1016/j.solener. 2015.12 .027

Bahaj, A.S., James, P.A., Jentsch, M.F., 2008. Potential of emerging glazing technologies for highly glazed buildings in hot arid climates. Energy and Buildings 40, 720-731.

Bird, R.E., 1984. A simple, solar spectral model for direct-normal and diffuse horizontal irradiance. Solar energy $32,461-471$.

Bird, R.E., Hulstrom, R.L., Lewis, L., 1983. Terrestrial solar spectral data sets. Solar energy 30, 563-573.

Bohren, C., Huffman, D., 1983. Absorption and scattering of light by small particles. Wiley Interscience $13,123-127$.

Chow, T., Fong, K., He, W., Lin, Z., Chan, A., 2007. Performance evaluation of a PV ventilated window applying to office building of Hong Kong. Energy and Buildings 39, 643 - 650. 
Chow, T.t., Li, C., Lin, Z., 2010. Innovative solar windows for cooling-demand climate. Solar Energy Materials and Solar Cells 94, 212-220.

Chow, T.T., Li, C., Lin, Z., 2011. The function of solar absorbing window as water-heating device. Building and Environment 46, 955 - 960. doi:http://dx.doi.org/10.1016/j.buildenv.2010.10.027.

Corporation, L.R., 2008. Tracepro suite of optical and illumination design software. Lambda Research Corporation .

Cuce, E., Cuce, P.M., 2016. Vacuum glazing for highly insulating windows: Recent developments and future prospects. Renewable and Sustainable Energy Reviews 54, 1345 - 1357.

DOE, 2010. Energyplus engineering reference. The reference to EnergyPlus calculations .

Edwards, D., 1977. Solar absorption by each element in an absorber-coverglass array. Solar Energy 19, 401-402.

EN 410:2011, 2003. Glass in building. determination of luminous and solar characteristics of glazing .

Fang, Y., Eames, P.C., Norton, B., Hyde, T.J., Zhao, J., Wang, J., Huang, Y., 2007. Low emittance coatings and the thermal performance of vacuum glazing. Solar Energy 81, $8-12$.

Fang, Y., Hyde, T.J., Hewitt, N., 2010. Predicted thermal performance of triple vacuum glazing. Solar Energy 84, $2132-2139$.

Finlayson, E., Arasteh, D., Huizenga, C., Rubin, M., Reilly, M., 1993. WINDOW 4.0: Documentation of calculation procedures. Lawrence Berkeley Laboratory, University of California.

Furler, R., 1991. Angular dependence of optical properties of homogeneous glasses. Ashrae Transactions $97,1129-1133$.

Gardiner, D., Morris, S., Coles, H., 2009. High-efficiency multistable switchable glazing using smectic a liquid crystals. Solar Energy Materials and Solar Cells 93, 301 - 306. doi:http://dx.doi.org/10.1016/j. solmat.2008.10.023

Goia, F., 2012. Thermo-physical behaviour and energy performance assessment of PCM glazing system configurations: A numerical analysis. Frontiers of Architectural Research 1, $341-347$.

Goia, F., Zinzi, M., Carnielo, E., Serra, V., 2012. Characterization of the optical properties of a PCM glazing system. Energy Procedia 30, 428 - 437. 1st International Conference on Solar Heating and Coolingfor Buildings and Industry (SHC 2012).

Gorgolis, G., Karamanis, D., 2016. Solar energy materials for glazing technologies. Solar Energy Materials and Solar Cells 144, $559-578$.

Granqvist, C., Lansåker, P., Mlyuka, N., Niklasson, G., Avendaño, E., 2009. Progress in chromogenics: New results for electrochromic and thermochromic materials and devices. Solar Energy Materials and Solar Cells 93, 2032 - 2039. doi:http://dx.doi.org/10.1016/j.solmat.2009.02.026. iME-8. 
Granqvist, C.G., 2008. Oxide electrochromics: Why, how, and whither. Solar Energy Materials and Solar Cells 92, 203-208.

Gueymard, C.A., duPont, W.C., 2009. Spectral effects on the transmittance, solar heat gain, and performance rating of glazing systems. Solar Energy 83, 940 - 953.

Hale, G.M., Querry, M.R., 1973. Optical constants of water in the 200-nm to 200- $\mu$ m wavelength region. Applied optics 12, 555-563.

Harbecke, B., 1986. Coherent and incoherent reflection and transmission of multilayer structures. Applied Physics B 39, 165-170.

Heavens, O., 1991. Optical Properties of Thin Solid Films. Dover.

Howell, J.R., Siegel, R., Menguc, M.P., 2010. Thermal radiation heat transfer. CRC press.

Hulstrom, R., Bird, R., Riordan, C., 1985. Spectral solar irradiance data sets for selected terrestrial conditions. Solar Cells 15, 365-391.

Hutchins, M., Topping, A., Anderson, C., Olive, F., Van Nijnatten, P., Polato, P., Roos, A., Rubin, M., 2001. Measurement and prediction of angle-dependent optical properties of coated glass products: results of an inter-laboratory comparison of spectral transmittance and reflectance. Thin Solid Films $392,269-275$.

Hutchins, M.G., Ageorges, P., 1993. Angular-dependent spectral optical properties of architectural glazings: results of an interlaboratory comparison of measurements, in: SPIE's 1993 International Symposium on Optics, Imaging, and Instrumentation, International Society for Optics and Photonics. pp. $13-24$.

Ismail, K., Henrıquez, J., 2003. Modeling and simulation of a simple glass window. Solar energy materials and solar cells $80,355-374$.

ISO 15099, 2003. Calculating center-glass performance indices of windows .

ISO 9050, 2012. Glass in building - determination of light transmittance, solar direct transmittance, total solar energy transmittance, ultraviolet transmittance and related glazing factors .

Jelle, B.P., Hynd, A., Gustavsen, A., Arasteh, D., Goudey, H., Hart, R., 2012. Fenestration of today and tomorrow: A state-of-the-art review and future research opportunities. Solar Energy Materials and Solar Cells 96, 1-28.

Jonasz, M., Fournier, G., 2011. Light Scattering by Particles in Water: Theoretical and Experimental Foundations: Theoretical and Experimental Foundations. Academic Press.

Karlsson, J., Karlsson, B., Roos, A., 2001. A simple model for assessing the energy performance of windows. Energy and Buildings 33, $641-651$.

Klainsek, J.C., 1991. Glazing and its influence on building energy behavior. Renewable energy 1, 441-448. 
Lechowska, A., 2016. A $\{$ CFD $\}$ study and measurements of double glazing thermal transmittance under downward heat flow conditions. Energy and Buildings 122, 107 - 119. doi:http://dx.doi.org/10.1016/ j.enbuild.2016.04.023

Liao, W., Xu, S., 2015. Energy performance comparison among see-through amorphous-silicon pv (photovoltaic) glazings and traditional glazings under different architectural conditions in china. Energy $83,267-275$.

Machado, M., Baenas, T., Yurrita, N., 2016. Optical model for multilayer glazing systems: Experimental validation through the analytical prediction of encapsulation-induced variation of $\{\mathrm{PV}\}$ modules efficiency. Solar Energy 135, 77 - 83. doi http://dx.doi.org/10.1016/j.solener.2016.05.040.

Maestre, I.R., Molina, J.L., Roos, A., Coronel, J.F., 2007. A single-thin-film model for the angle dependent optical properties of coated glazings. Solar Energy 81, 969-976.

McCluney, W.R., 2014. Introduction to radiometry and photometry. Artech House.

van Nijnatten, P.A., 1994. Mathematical modeling of optical glazing performance, in: Optical Materials Technology for Energy Efficiency and Solar Energy Conversion XIII, International Society for Optics and Photonics. pp. 753-762.

van Nijnatten, P.A., 1999. Predictive algorithms for directional optical properties of coated glazing for building and automotive applications. Thin Solid Films 351, 295 - 300.

Pfrommer, P., Lomas, K., Seale, C., Kupke, C., 1995. The radiation transfer through coated and tinted glazing. Solar Energy 54, 287-299.

Press, W., Teukolsky, S., Vetterling, W., Flannery, B., 2007. Numerical recipes: The art of scientific computing, 3rd éd.

Qahtan, A., Rao, S., Keumala, N., 2014. The effectiveness of the sustainable flowing water film in improving the solar-optical properties of glazing in the tropics. Energy and Buildings 77, $247-255$. doi:http://dx.doi.org/10.1016/j.enbuild.2014.03.051.

Roos, A., Polato, P., Van Nijnatten, P.A., Hutchins, M.G., Olive, F., Anderson, C., 2001. Angulardependent optical properties of low-e and solar control windows: Simulations versus measurements. Solar Energy 69, 15-26.

Rubin, M., 1982. Solar optical properties of windows. International Journal of Energy Research 6, $123-133$.

Rubin, M., Powles, R., Rottkay, K.V., 1999. Models for the angle-dependent optical properties of coated glazing materials. Solar Energy 66, $267-276$.

Rubin, M., Von Rottkay, K., Powles, R., 1998. Window optics. Solar energy 62, 149-161.

Shurcliff, W.A., 1974. Transmittance and reflection loss of multi-plate planar window of a solar-radiation collector: Formulas and tabulations of results for the case n=1. 5. Solar Energy 16, 149-154. 
Siegel, R., 1973. Net radiation method for transmission through partially transparent plates. Solar Energy $15,273-276$.

Sierra, P., Hernández, J.A., 2017. Solar heat gain coefficient of water flow glazings. Energy and Buildings 139, 133 - 145. doi:http://dx.doi.org/10.1016/j.enbuild.2017.01.032.

Silva, T., Vicente, R., Rodrigues, F., 2016. Literature review on the use of phase change materials in glazing and shading solutions. Renewable and Sustainable Energy Reviews 53, 515 - 535.

Stenzel, O., 2005. The Physics of thin film optical spectra. Springer.

Suárez, M.J., Gutiérrez, A.J., Pistono, J., Blanco, E., 2011. CFD analysis of heat collection in a glazed gallery. Energy and Buildings 43, 108 - 116.

Tina, G., Gagliano, A., Nocera, F., Patania, F., 2013. Photovoltaic glazing: Analysis of thermal behavior and indoor comfort. Energy Procedia 42, 367 - 376. Mediterranean Green Energy Forum 2013: Proceedings of an International Conference MGEF-13.

Vera, J.T., Laukkanen, T., Sirén, K., 2014. Multi-objective optimization of hybrid photovoltaic-thermal collectors integrated in a $\{$ DHW $\}$ heating system. Energy and Buildings 74, 78 - 90. doi:http://dx. doi.org/10.1016/j.enbuild.2014.01.011.

Walton, G.N., 1986. Modeling window optics for building energy analysis. Technical Report. National Bureau of Standards, Washington, DC (USA). Building Physics Div.

Wijeysundera, N., 1975. A net radiation method for the transmittance and absorptivity of a series of parallel regions. Solar Energy 17, 75-77.

Wright, J.L., 1998. Calculating center-glass performance indices of windows. Transactions - American Society of Heating Refrigerating and Air Conditioning Engineers 104, 1230-1244.

\section{AppendixA. Inverse problem for uncoated glass or PVB layer}

An uncoated glass or PVB layer is characterized by the refractive index $n$ and the extinction coefficient $\kappa$. The IGBD provides the values for transmittance $T$ and reflectance $R$ of a layer embedded in air at normal incidence. The objective of the inverse problem is to determine $n$ and $\kappa$ from the transmittance $T$ and reflectance $R$. Due to the symmetry of the problem, $R=R^{\prime}$ and the spectral properties of the left and right interfaces coincide. When considering an uncoated glass or PVB layer no radiation is absorbed and the fraction of the incident radiation which is reflected is $r_{1}=r_{2}=\rho$. Consequently, the transmissivity of the interface is $t_{1}=t_{2}=1-\rho$. Note that $r_{1}^{\prime}$ and $r_{2}^{\prime}$ are equal to $\rho$ due to the symmetry of the problem. These equations are equivalent to those encountered in McCluney (2014). Particularizing these values in equations $31-33$ :

$$
\begin{aligned}
T & =\frac{(1-\rho)^{2} \tau}{1-\rho^{2} \tau^{2}} \\
R & =\rho+\frac{(1-\rho)^{2} \rho \tau^{2}}{1-\rho^{2} \tau^{2}}
\end{aligned}
$$


An intermediate step in the inverse problem (Furler, 1991) consists of obtaining $\tau$ and $\rho$ from equations A.1 and A.2:

$$
\begin{gathered}
\rho=\frac{\left(T^{2}-R^{2}+2 R+1\right)-\sqrt{\left(T^{2}-R^{2}+2 R+1\right)^{2}-4 R(2-R)}}{2(2-R)}, \\
\tau=\frac{R-\rho}{\rho T} .
\end{gathered}
$$

From these values, the refractive index $n$ and the extinction coefficient $\kappa$, for a layer of thickness $d$ and considering normal incidence in equations (35) and (36), are:

$$
\begin{gathered}
n=\frac{1+\sqrt{\rho}}{1-\sqrt{\rho}}, \\
\kappa=-\frac{\lambda}{4 \pi d} \ln \tau .
\end{gathered}
$$

\section{AppendixB. Inverse problem for coated glass at left interface}

For coated glass panes, the IGDB provides the transmittance $T$, the front reflectance $R$ and the back reflectance $R^{\prime}$ of the coating deposited on a substrate. The left interface where the coating is deposited can be characterized by its transmittance $t$, reflectance $r$ and back reflectance $r^{\prime}$, as explained in subsection 5.2. In this case, the inverse problem consists of determining the properties of the interface: $t, r, r^{\prime}$ where the coating is located from the measurements of the coated glass pane and the substrate. It is assumed for this inverse problem that the coating is deposited at the left interface. Hence, for the coated interface $t_{1}=t, r_{1}=r, r_{1}^{\prime}=r^{\prime}$, and for the uncoated interface (see subsection 5.1) $t_{2}=1-\rho, r_{2}=r_{2}^{\prime}=\rho$. Particularizing these values in equations 31)-333:

$$
\begin{aligned}
T & =\frac{t(1-\rho) \tau}{1-\rho r^{\prime} \tau^{2}}, \\
R & =r+\frac{t^{2} \rho \tau^{2}}{1-\rho r^{\prime} \tau^{2}}, \\
R^{\prime} & =\rho+\frac{(1-\rho)^{2} \tau^{2} r^{\prime}}{1-\rho r^{\prime} \tau^{2}} .
\end{aligned}
$$

One possible way to obtain the properties $t, r, r^{\prime}$ of the coated interface is from equations B.1 B.3. However, it is required to know the transmissivity $\tau$ and reflectivity $\rho$ of the substrate as a function of wavelength and angle of incidence. Once the refractive index and the extinction coefficient of the substrate are known, the prodedure is indicated in subsection 5.1. Note that the substrates used by manufacturers are shared between several coatings. In the IGDB, the coated glass information contains the reference to the substrate. In this case, the properties of the interface are determined:

$$
\begin{aligned}
t & =\frac{T(1-\rho)}{\tau\left(1-2 \rho+R^{\prime} \rho\right)}, \\
r & =R-\frac{T^{2} \rho}{1-2 \rho+R^{\prime} \rho}, \\
r^{\prime} & =\frac{R^{\prime}-\rho}{\tau^{2}\left(1-2 \rho+R^{\prime} \rho\right)} .
\end{aligned}
$$

